\title{
Intermédialités
}

Histoire et théorie des arts, des lettres et des techniques

Intermediality

History and Theory of the Arts, Literature and Technologies

\section{Nouvelles formes d'art et d'expérience esthétique dans une culture en transit : les promenades de Janet Cardiff}

\section{Walter Moser}

Numéro 15, printemps 2010

exposer

displaying

URI : https://id.erudit.org/iderudit/044684ar

DOI : https://doi.org/10.7202/044684ar

Aller au sommaire du numéro

Éditeur(s)

Revue Intermédialités (Presses de l’Université de Montréal)

ISSN

1705-8546 (imprimé)

1920-3136 (numérique)

Découvrir la revue

Citer cet article

Moser, W. (2010). Nouvelles formes d'art et d'expérience esthétique dans une culture en transit : les promenades de Janet Cardiff. Intermédialités /

Intermediality, (15), 231-250. https://doi.org/10.7202/044684ar
Résumé de l'article

Cette étude part du constat que notre condition culturelle contemporaine est marquée par l'augmentation et l'intensification des mobilités à la fois physiques (locomotion) et médiatiques (médiamotion). En interaction avec cette condition, de nouvelles formes d'art émergent. L'analyse porte sur une de ces nouvelles formes réalisée par l'artiste canadienne Janet Cardiff dans le genre des audio-visual walks, et plus particulièrement sur Conspiracy Theory créé en 2002 à l'occasion d'une exposition au MAC de Montréal. Elle se concentre sur la sculpture sonore de la bande auditive et les dispositifs d'énonciation visuelle de l'image vidéo afin de comprendre la manière dont cette oeuvre détermine une expérience esthétique de la mobilité pour le promeneur déambulatoire. Tout en s'en alimentant, cette expérience aménage un espace cognitif et critique par rapport à la culture ambiante. 


\title{
Nouvelles formes d'art et d'expérience esthétique dans une culture en transit: les promenades de Janet Cardiff
}

\author{
Walter Moser
}

e soutiendrai ici qu'une des caractéristiques de la situation culturelle contemporaine réside dans sa grande mobilité et instabilité, et que certaines nouvelles formes d'art et des modalités de création artistiques captent, reflètent et donnent à penser cette caractéristique de notre culture.

Je propose la métaphore épistémique de «culture en transit» pour désigner la dynamique culturelle dont nous faisons l'expérience quotidiennement, et qui a comme contrepartie une fragilisation des cultures traditionnelles et des identités établies. Tributaire de ce qu'on désigne communément aujourd'hui par «mondialisation », cette dynamique-et-fragilité, mobilité-et-instabilité s'accentue sous l'impact d'un ensemble complexe de facteurs, dont les flux migratoires, la logique économique capitaliste, la commercialisation de la culture, les restructurations politiques, les nouvelles technologies de télécommunication et d'archivage et les nouveaux médias.

Pour des besoins d'analyse, j’identifierai deux types de mouvements participant de cette culture en transit: la locomotion et la médiamotion ${ }^{1}$. Le terme de «locomotion » réunit l'ensemble des phénomènes et impacts culturels provoqués par la grande mobilité des personnes dans le monde contemporain, qu'il s'agisse de mobilité librement choisie ou forcée. Déplacement, dislocation, migration, fuite: il est question ici en premier lieu du mouvement physique des êtres

1. J'ai élaboré ce cadre théorique dans mon texte «La culture en transit: locomotion, médiamotion, artmotion », Gragoatá, n 17, 2004, p. 25-41. 
humains. Ce mouvement a toujours existé, mais il paraît évident qu'il a sensiblement augmenté et s'est intensifié dans le monde contemporain.

On réunira sous le terme de «médiamotion» l'ensemble des mouvements qui ont leur moteur dans les médias. De nos jours, la situation type au quotidien étant une personne assise devant un écran, le corps humain peut rester immobile: c'est le monde médiatisé qui est en mouvement sur l'écran et qui peut être capté, en médiation symbolique, par l'appareil sensoriel de l'être humain. Le «multimédia », sur support numérique, a aujourd'hui intensifié ce type de mobilité qui n’était, cependant, pas étranger à des médias plus traditionnels : livre, presse écrite, téléphone ou radio. Il procure des effets paradoxaux : le contact à distance, l'immédiateté de ce qui est médiatisé, l’instantanéité de l'ici et là.

Ces deux types de mobilité font partie de la situation culturelle contemporaine, la déterminent même à certains égards, et se combinent dans bien des contextes quotidiens: tout automobiliste roulant avec la radio allumée, tout flâneur métropolitain (surtout celui muni d'un baladeur, d'un iPod et/ou d'un téléphone cellulaire) participe aujourd'hui de ces deux types de mouvement. En parallèle, dans la production artistique, nous assistons aujourd'hui au développement de formes et de genres artistiques qui se trouvent dans une relation d'interaction et de com-possibilité avec la culture en transit. Je pense en particulier à certaines formes de performances, et d'installations, par exemple à certaines œuvres (le terme est-il encore adéquat?) de Jana Sterbak, de Gary Hill, de Bill Viola, de Shirin Neshat, et de bien d'autres.

\section{LES PROMENADES DE JANET CARDIFF}

Mais je pense plus particulièrement à un genre nouveau, développé il y a une vingtaine d'années par l'artiste canadienne Janet Cardiff, en collaboration avec George Bures Miller : les «promenades» (walks) sur lesquelles je me concentrerai dans ce qui suit. Janet Cardiff n’a pas créé que des «promenades », loin de là : elle est également connue pour des installations telles que The Whispering Room (1991), To Touch (1993), The Dark Pool (1995), Fourty Part Motet (2001), The Paradise Institute (2001) et plus récemment The Killing Machine (2007)2. C'est en 1991 qu'elle a commencé à développer une forme d'œuvre d'art tout à fait originale: le walk ou walking piece, appelé «promenade» en français. Voici comment Carolyn Christov-Barkagiev décrit ce nouveau genre artistique:

2. The Whispering Room et Fourty Part Motet, installations sonores, comportent déjà l'élément de la déambulation, mais la médiation technique du son est fixe (haut-parleurs sur tiges) et on se «promène » dans le lieu restreint de l'installation; l'accès est collectif. 
Cardiff's walks are time-based fictional works that are experienced by participants who don a headset attached to a Discman or DV Walkman and follow pre-recorded instructions that lead them into open-ended and ambiguous narratives. Her own voice features prominently in these haunting works as she guides her audience on routes that take them to unexpected places both indoors and out ${ }^{3}$.

Janet Cardiff a commencé sa production en 1991, avec des promenades exclusivement auditives: un promeneur équipé d'une espèce de baladeur reçoit des écouteurs alimentés par une bande sonore portable. Guidé par la voix de Janet Cardiff enregistrée sur ce support médiatique, il fait une promenade dont le déroulement et le parcours sont pré-programmés. Ainsi combine-t-il la déambulation dans un site réel en temps réel, site qui offre son propre bruitage, avec l'écoute de la bande sonore qui, en plus de le guider, l'enveloppe dans un monde sonore fictionnel d'une grande intensité et complexité. L'artiste a choisi des sites dans plusieurs pays pour ses promenades:

$\begin{array}{ll}1991 & \text { Forest Walk (Banff, Canada) } \\ 1991 & \text { Bathroom Stories (Lethbridge, Canada) } \\ 1992 & \text { An Inability to Make a Sound (Halifax, Canada) } \\ 1996 & \text { Louisiana Walk (Danemark) } \\ 1996 & \text { I've Been Waiting for You (Los Angeles, États-Unis) } \\ 1997 & \text { Münster Walk (Münster, Allemagne) } \\ 1997 & \text { Chiaroscuro I (San Franciso, États-Unis) } \\ 1998 & \text { Villa Medici Walk (Rome, Italie) } \\ 1998 & \text { Wanas Walk (Knislinge, Suède) } \\ 1998 & \text { Drogan's Nightmare (São Paulo, Brésil) } \\ 1998 & \text { Mallin's Night Walk (Pound Ridge, New York, États-Unis) } \\ 1999 & \text { Missing Voice (Case Study B) (Londres, Grande-Bretagne) } \\ 1999 & \text { MoMA Walk (New York, États-Unis) } \\ 1999 & \text { Waterside Walk (Harmondsworth, Grande-Bretagne) } \\ 1999 & \text { Sleepwalking (New York, États-Unis) } \\ 1999 & \text { In Real Time (premier audio-visual Walk) (Pittsburg, États-Unis) } \\ 2000 & \text { A Large Slow River (Ontario, Canada) }\end{array}$

3. Carolyn Christov-Bakargiev, Janet Cardiff. A Survey of Works Including Collaborations with George Bures Miller, New York, P.S. 1 Contemporary Art Center, 2001, p. 15. On consultera également le livre (avec CD) de Janet Cardiff, The Walk Book, Cologne, Walther König, 2005, et le film documentaire de Wiebke von Carolsfeld: Walk with us. The Collaborative Works of Janet Cardiff and George Bures Miller, 2005. 


$\begin{array}{ll}2000 & \text { Taking Pictures (St. Louis, États-Unis) } \\ 2001 & \text { The Telephone Call (San Francisco, États-Unis) } \\ 2001 & \text { P.S. 1 Walk (New York, États-Unis) } \\ 2001 & \text { Ittingen Walk (Warth, Suisse) } \\ 2002 & \text { Conspiracy Theory (Montréal, Canada) } \\ 2004 & \text { Her Long Black Hair (New York, États-Unis) } \\ 2005 & \text { Ghost Machine (Berlin, Allemagne) } \\ 2005 & \text { Words Drawn in Water (Washington, États-Unis) } \\ 2006 & \text { Jena Walk (Memory Field) (Jena, Allemagne) }\end{array}$

À partir de 1996, elle a commencé à complexifier les promenades en y ajoutant une bande vidéo. Le promeneur est alors muni, en plus des écouteurs, d'un appareil vidéo numérique dont le moniteur offre une image mobile tout au long de la promenade. Une partie des didascalies pour guider le promeneur est désormais transférée du média sonore au média visuel.

Dans ce qui suit, je m'intéresse à ce genre artistique et plus particulièrement à l'expérience spécifique qu'il procure au « promeneur $^{5} »$. Mais je me trouve, pour ce faire, assez démuni, et ceci pour deux raisons, dont la première est plus «classique » que la seconde : c'est que l'expérience esthétique, dans son noyau, est rigoureusement personnelle et individuelle, même si elle est faite dans des lieux publics $^{6}$. Et la rapporter pose un problème déjà bien connu et débattu sous la catégorie de l'expression: comment extérioriser et rendre socialement accessible et "partageable », en se servant d'un système de signes façonné par et appartenant à une collectivité, ce qui relève de l'intériorité subjective? Certes, cette expérience se trouve toujours insérée dans un contexte culturel où règnent des goûts esthétiques relevant du collectif, mais cela ne rend pas moins ardue la verbalisation d'une expérience largement non verbale.

4. Ce déplacement des walks, et donc de l'artiste qui les crée, indique la dimension globalisante du genre: le site est rigoureusement local mais il se multiplie à travers le monde. Si je veux faire l'expérience de ces différents walks, je dois me déplacer. Aucune reproduction médiale ne peut en rendre compte adéquatement, puisque ma déambulation in situ fait partie de l'œuvre. Par la suite, je me concentrerai sur cet autre type de déplacement qu'est la déambulation même, prototype de la locomotion.

5. Je qualifierai d'esthétique cette expérience de l'œuvre d'art, sans pour autant charger ce terme de tout le débat philosophique dont il a déjà fait l'objet.

6. Ceci est le cas a fortiori avec les promenades audio et vidéo, car l'accès à l'œuvre est rigoureusement limité à une personne à la fois, contrairement à des expériences esthétiques qu'on peut faire collectivement, y compris une visite au musée d'art. 
La seconde raison est plus spécifique aux promenades de Janet Cardiff. C'est que l'artiste a donné des instructions sur la manière de faire les promenades: elles doivent se dérouler sans interruption, et sans qu'il y ait séparation entre la bande sonore et la bande vidéo, même pas pour ceux qui, comme moi, veulent en faire l'analyse! Pour qu'on puisse tout de même rendre compte des promenades sans en faire l'expérience, elle a autorisé, dans le cas de Théorie du complot (Conspiracy Theory, 2002), la publication d'une série de diapositives. Ce sont des photos prises pendant que quelqu'un fait la promenade, dans une perspective de trois quarts de dos et par-dessus son épaule. Nous avons «le droit» de visionner l'enregistrement de quelqu'un en train de faire la promenade, in situ, et donc de vivre l'expérience visée ici, mais nous n'avons pas le droit d'en décomposer les parties, de dissocier les divers éléments dont la synchronisation fait l'œuvre.

Adoptant une attitude analytique, essayons tout de même d'identifier quelques-uns de ces éléments afin de pouvoir comprendre leur interaction dans la production d'une expérience esthétique très particulière.

Le genre artistique de la promenade combine deux aspects de notre expérience culturelle plus générale: la locomotion et la médiamotion. Je me déplace dans un lieu réel qui a été sélectionné par l'artiste, et selon un parcours programmé par elle. Ma déambulation est un élément constitutif de l'œuvre ${ }^{7}$, mon corps en déplacement devient un matériau au même titre que le son ou les images enregistrés et rejoués ${ }^{8}$. Aussi le déplacement de mon corps, selon un rythme prescrit par l'artiste, fait-il partie intégrante de mon expérience esthétique, ce qui n’est pas le cas de ma déambulation dans une pinacothèque.

On ne saurait sous-estimer cet aspect qui peut paraître anodin : Janet Cardiff permet au sujet de faire une expérience esthétique, de jouir esthétiquement sans être séparé du monde réel, sans être immobilisé - littéralement ou métaphoriquement - dans une attitude de recueillement et de contemplation, sans être

7. Cette déambulation, surtout en milieu urbain, rappelle la figure du flâneur baudelairien. Isabelle Rieusset-Lemarié parle de «la pratique déterminante de la déambulation» en se référant à Walter Benjamin: Isabelle Rieusset-Lemarié, La société des clones. À l'ère de la reproduction multimédia, Paris, Actes Sud, 1999, p. 364.

8. Voir la classification et la réflexion englobante qu'offre Monika Wagner dans son livre Das Material der Kunst. Eine andere Geschichte der Moderne, Munich, C. H. Beck, 2001. En transposant le terme allemand «Material» en français, il ne faut, cependant, pas oublier que la langue française y introduit une différence non négligeable, car elle distingue entre matériau et matériel. Monika Wagner travaille davantage dans l'aire sémantique du concept de «matériel». 
attaché au préalable au mat comme Ulysse 9 . Plus encore, elle intègre la participation libre du corps sous la forme d'un déplacement dans l'espace (loco-motion) comme une composante constitutive dans son œuvre et dans l'expérience que je peux en faire.

Précisons que, traditionnellement, certaines œuvres artistiques réalisées dans les trois dimensions de l'espace - on pense aux œuvres architecturales, sculpturales, surtout les sculptures géantes de Richard Serra - demandent également un déplacement physique pour être perçues au complet. Mais ce type de locomotion esthétique n'est pas constitutivement accompagné d'un élément de médiamotion, comme c'est le cas avec les walks. Par ailleurs l'exercice de la promenade, comme celui rapporté par Rousseau dans Les rêveries du promeneur solitaire (1778) - qu'on peut verser à la pré-histoire des walks de Janet Cardiff - ne constitue pas en soi une expérience esthétique.

L'autre mouvement dont je fais l'expérience simultanément est celui qui est enregistré sur la bande sonore, et dans certains cas aussi sur la bande vidéo. La bande sonore, produite grâce à la technologie la plus avancée selon des méthodes d'enregistrement auxquelles je reviendrai, nous propose divers éléments dont l'interaction est d'une remarquable complexité : la voix principale et dominante est celle de Janet Cardiff elle-même. C’est une voix calme, agréable, veloutée, un peu sottovoce ${ }^{10}$ et pourtant envoûtante. Elle s'affirme comme une voix intérieure, intime qui raconte, réfléchit et crée un espace de communication privé qui ensorcelle le promeneur jusqu'au point d'obtenir un effet de transfert sonore (est-ce elle, est-ce moi?). On est comme enfermé dans l'espace sonore de cette voix. On s'y habitue, on l'attend après ses interruptions, on la suit: c'est elle qui donne la plupart des instructions sur la «marche à suivre», sans être insistante ou impérieuse toutefois: go down the stairs, turn right, open the door...

Selon la promenade considérée, une ou plusieurs autres voix s'y ajoutent, masculines et féminines. Mais c'est la voix de Janet Cardiff qui nous accompagne tout au long, nous guide à travers la promenade, nous introduit dans un univers fictionnel par des bouts de récits, toujours interrompus et laissés ouverts et indécis, par des évocations du passé qui relèvent du lieu et du personnage que la voix incarne, par des fragments de rêve. Entremêlés avec cette voix, dans un montage parfois dur et abrupt, surgissent des bruitages, des capsules sonores (coups de feu, moteur à plein régime, bruit d’hélicoptère...), mais aussi

9. Voir l'analyse - anachronique à dessein! - que proposent Horkheimer et Adorno de l'épisode du chant des Sirènes dans l'Odyssée, dans leur Dialectique de la raison.

10. Les commentaires en anglais parlent d'un flat tone. 
des allusions sonores à des films, ou d'autres fragments tirés de notre répertoire sonore culturel, qui nous déplacent dans d'autres lieux et temps et qui créent souvent un grand contraste avec le lieu réel de la promenade. C'est dans ces évocations médiatiques d’autres espaces-temps que s’active la dimension «médiamotion» de la promenade.

On a dit que Janet Cardiff est une véritable sculptrice du matériau son. Elle a fait de l'auditif son terrain et matériau premier de travail. Avec cela, elle a opté pour un sens de la proximité, qui favorise l'intimité, et relève socialement souvent du privé, tout en donnant lieu au secret, à la rumeur, au complot (le bouche à oreille!), comme l'a bien remarqué l'historienne de l'art Marie Fraser dans un colloque à Montréal ${ }^{11}$.

J'enchaîne ici avec une digression d'histoire culturelle qui permettra de situer le travail des promenades dans un plus vaste contexte que celui de «l'art contemporain ». C'est que, sculptrice du son, Janet Cardiff participe d'une réorientation importante et profonde de notre «aïsthésis » culturelle. Après une hégémonie de l'écrit et de l'imprimé, après une longue domination de la vue comme la perception sensorielle primordiale, que d'aucuns ont baptisée «oculocentrisme ${ }^{12}$ ", elle concourt à la redécouverte et à la réhabilitation du sens de l'ouïe et d'une oralité qu'on ne saurait accuser de «phonocentrisme». Son travail n’est pas porté, ni affectivement ni conceptuellement, par la nostalgie d'une oralité originaire et plénière. Mais cette réactivation du matériau sonore et de l'ouïe comme organe sensoriel, elle la réalise d'emblée à travers les médias et les technologies d'enregistrement, de reproduction et de transmission les plus modernes. Elle se situe donc résolument dans une «secondary orality» (Walter Ong) et accepte l'existence de l'appareillage médiatico-technologique comme faisant intégralement partie de la condition contemporaine. Plus que de l'accepter, comme par résignation, comme une contrainte se soldant par une perte, ou comme un pis aller par rapport à un idéal perdu, elle y reconnaît un potentiel esthétique et en explore systématiquement les possibilités.

Elle se situe par là dans la foulée de l'espèce de révolution bioanthropologique que proposait Herder dans ses Ideen zur Philosophie der Geschichte der

11. Territoires de l'intermédialité et géographies sonores. Une réflexion à partir des Promenades de Janet Cardiff, dans le cadre du cinquième colloque du Centre de recherche sur l'intermédialité (CRI), La nouvelle sphère intermédiatique V. Histoire et géographie d'un concept. L'intermédialité entre les savoirs, Fondation Daniel Langlois et Goethe Institut, du $1^{\text {er }}$ au 4 octobre 2003.

12. Voir Martin Jay, Downcast Eyes: The Denigration of Vision in Twentieth-Century French Thought, Berkeley et Los Angeles, University of California Press, 1993. 
Menschheit (1784-1791) en délogeant le sens de la vue de sa position dominante et en y instaurant le sens de l'ouïe comme le sens humain par excellence. Il argumentait $^{13}$ que l'être humain est un Mittelgeschöpf (une créature du milieu, un être de l'entre-deux, une espèce destinée à la médiation), ce qui plaçait automatiquement le sens de moyenne portée, l'ouie, au centre de ce qui définissait son être dans le monde matériel et biologique ${ }^{14}$.

Dans une entrevue imprimée dans le catalogue, Janet Cardiff semble continuer cette réflexion bioanthropologique de Herder en la tirant cependant davantage du côté de la pénétration de l'espace intérieur (psychique, affectif, imaginaire et cognitif) par le matériau auditif:

Conceptually, I was doing the same thing in the eighties that I am now. Mixing, layering ideas, making narrative juxtapositions. Only I was doing it visually. Visually, though, you didn't really get it directly somehow. There is something about audio that goes right into you directly. You can't stop it. Your brain doesn't cut it off. Your brain has more of a filtering process for visual information, I think, than with the audio. I don't know why it is, but somehow sound just comes into your subconscious much faster and easier. As on the soundtracks, there is something interesting that happens when I relate my memories, something about time slippage. When I'm talking about my memories, they become immediate for the listener. For me, they'll always be past, but for the listener, they are part of the present. I'm interested in working with time in this way, flip-flopping between past and present, using memory and bringing it into the present ${ }^{15}$.

Contrairement au dicton qui fait des yeux les fenêtres de l'âme, ici l'accès le plus direct à l'âme semble être l'oreille. Janet Cardiff utilise le sens de l'ouïe, en fait, comme le sens de la proximité et construit, à l'aide de la bande sonore, l'icimaintenant d'un espace-temps que partagent alors le promeneur et la voix féminine qui le guide. Cette construction est médiatique; elle s'ajoute et se superpose

13. Tout en s'appuyant encore sur la figure traditionnelle de The Great Chain of Being (voir le livre du même titre par Arthur O. Lovejoy, 1936) qui établit une hiérarchie continue entre tous les êtres existants.

14. «Esthésiquement» parlant, cette position du milieu attribuée par Herder à l'être humain a comme conséquence le fait que l'ouïe devient son sens principal. La vue est le sens divin par excellence; les sens plus grossiers tels que le tact et le goût, plus animaux, sont des sens de l'immédiateté; ils exigent un contact matériel-corporel direct avec le monde environnant et entraînent un rétrécissement de ce dernier. Herder offre ainsi une version bioanthropologique d'une re-théorisation des sens, très répandue au $18^{\mathrm{e}}$ siècle, que d'autres auteurs abordent d'un point de vue gnoséologique (Diderot, Condillac) et esthétique (Kant).

15. Christov-Bakargiev, 2001, p. 27. 
au soundscape «naturel », et par là non contrôlé, du lieu réel de la promenade. Ce double ici-maintenant subit, par moments, l'irruption d'un ailleurs sonore qui peut être violent et complexifie encore la stratification sonore ${ }^{16}$ résultant d'un effet de médiamotion comme nous le vivons quotidiennement, surtout en milieu urbain.

Si une part de l'expérience esthétique du promeneur dépend des contingences du lieu de la promenade, l'autre part est rigoureusement programmée, calculée et exécutée avec la plus grande sophistication technologique. Afin de capter et d'exploiter au maximum la force suggestive du son, Janet Cardiff, conjointement avec George Bures Miller, a en fait développé des techniques d'enregistrement très particulières :

With binaural recording, it is possible to suggest 3-D space, and therefore the presence of physical phenomena that aren't actually there. Binaural recording is achieved by placing two omni-directional microphones in the position of the ears on a dummy head, and recording in stereo while moving the head through space. Played back on a headset, the sounds seem to come from the actual surrounding. Subconsciously, participants begin to breathe and walk in synch with the virtual body on the tape or $\mathrm{CD}$, blurring the distinction between self and other ${ }^{17}$.

Je peux en témoigner: l'hyperréalité du son, infusé de la sorte dans notre ouïe, est si puissante qu'on commence à synchroniser le mouvement de notre déambulation avec les pas qui la rythment par distillation dans les oreilles. Cet effet corporellement réel de fusion, ou du moins de confusion entre le réel et ce que Janet Cardiff appelle ici le virtuel, trouve son équivalent et redoublement dans la structure d'énonciation du texte oral que la voix nous administre. En principe, la voix de Janet Cardiff appartient à une locutrice «I » qui s'adresse à un promeneur «you». Cette dissociation de deux instances est renforcée tout particulièrement par l'usage de l'impératif par lequel le promeneur est instruit sur le déroulement du parcours et guidé tout au long de celui-ci. Mais subrepticement, une coalescence des deux instances a lieu d'où émerge un «we » qui

16. Janet Cardiff parle en fait souvent de «layering of sound»; cette métaphore spatiale pour parler du travail avec le matériau sonore est en soi intéressante.

17. Christov-Bakargiev, 2001, p. 22. Voilà une des techniques; une autre est décrite comme suit: "The participant carrying the Walkman will be instructed by a voice on the tape to follow a specific route. This voice is recorded with the microphones close to the body while walking the actual site. This method causes the listener to feel as if the recorded body and voice are inside the listener's body and creates an intimate connection. Subconsciously, participants begin to breathe and walk in synch with the virtual body on the tape, blurring the distinction between self and other », ibid., p. 80. 
abolit la différence des interlocuteurs et par là l'espace d'interlocution. Cette manipulation langagière entrâne un effet d'identification qui agit même pardessus les barrières entre les genders, pourtant physiquement bien marquées dans l'organe de la voix. Toutefois, le déroulement de la promenade n'est pas inscrit dans une dynamique narrative qui irait de la dissociation vers la coalescence (étrangeté, séparation $=>$ identification, fusion), il se maintient, au contraire, dans un mouvement de va-et-vient - en transit - entre les deux positions.

Nonobstant ces effets de fusion et d'identification, le dispositif de la promenade comporte une structure de domination et de pouvoir que Janet Cardiff, en tant que femme, assume avec un malin plaisir et avec une intention critique certaine. C'est qu'elle lui permet de renverser la relation traditionnelle entre homme et femme:

... it may be a reaction to art history and to our culture's obsession with male desire. Think of analyzing Jackson Pollock - ideas of virility and genius related to virility. In my pieces it's the woman's voice that bosses you around, the voice of authority ${ }^{18}$.

Avant d'aborder l'ajout de la bande vidéo, un premier bilan s'impose. Combinant la déambulation d'un promeneur réel dans un lieu réel avec des déplacements suggérés auditivement moyennant un dispositif médiatique, le genre de la promenade combine en fait la locomotion avec la médiamotion, deux des principaux ingrédients de notre culture en transit. Ainsi, Janet Cardiff ne reproduit pas seulement des éléments de la culture environnante, mais elle confère à l'expérience esthétique que nous procure la promenade la structure d'un être en transit. Nous faisons l'expérience d'une mobilité-et-instabilité qui résulte d'un mouvement entre deux types de réalité. L'essentiel, c'est qu'on n'est pas acheminé d'une proposition de réalité vers une autre qui s'avérerait plus authentique, mais on reste entre deux propositions qui sollicitent notre corps, dans l'œuvre d'art, avec une égale intensité « esthésique».

Sur le plan de la perception et de la cognition, on est engagé, comme le dit le critique Ralph Rugoff que Janet Cardiff mentionne dans son entrevue ${ }^{19}$, dans une expérience schizophrénique. Mais cette schizophrénie n’est pas que perceptive. Une structure de l'indécision entre deux possibilités, du passage entre deux positions, du va-et-vient entre deux instances s'affirme comme ce qu'il y a de plus constant dans ce genre d'art original ${ }^{20}$. On reste en permanence en transit entre

18. Ibid., entrevue p. 22.

19. Ibid., p. 25.

20. Dans le texte anglais, tant de la critique Carolyn Christoph-Bakargiev que dans l'entrevue donnée par Janet Cardiff elle-même, on trouve un grand nombre de 
deux possibilités sans aboutir, et encore moins appartenir, en définitive ni à l'une ni à l'autre. La dynamique de cette structure «en transit» semble en fait être une des matrices structurelles les plus profondes de l'œuvre, et déterminer de la sorte l'expérience que nous en faisons.

Elle se manifeste à tous les niveaux de cette expérience: on doit négocier le va-et-vient entre le paysage réel et le paysage médiatique. On navigue entre le son du site réel et la bande vidéo. On passe des contingences incontrôlées du vrai lieu de la promenade à la pré-programmation médiatique d'une autre réalité, et vice-versa. On glisse d'une structure de domination à un effet d'identification qui se défait à son tour. L'association des voix alterne avec la dissociation des voix. L'insertion dans le lieu réel cède le pas au déplacement virtuel, ensuite le mouvement s'inverse. La mémoire du passé, qu'évoque le personnage qui nous accompagne aujourd'hui, est contenue et anticipée dans un rêve prémonitoire du personnage d'autrefois. Le passé se présentifie et le présent s'abîme dans le passé. Le promeneur qui se trouve sous la surveillance d'une caméra est celui-là même qui braque sa caméra vidéo sur les personnes qu’il domine de son regard.

\section{CONSPIRACY THEORY (2002)}

Dans cette promenade audio-vidéo, Janet Cardiff a fait de la propension de l'auditif, ce sens de la proximité, à l'intimité et au secret le thème principal même en l'intitulant Conspiracy Theory. Elle reprend ainsi une thématique que bien d'autres artistes travaillent aujourd'hui, dans différentes formes d'art, et qui exprime une appréhension ambiante assez généralisée qui est distillée, entre autres choses, par l'ubiquité des médias de surveillance. D'innombrables fois, elle a fait l'objet de fictions, surtout dans le cinéma ${ }^{21}$, pas seulement dans le film que Richard Donner a réalisé en 1997, et qui porte le même titre que la promenade de Janet Cardiff.

Cette pièce fut créée pour l'exposition rétrospective de l'œuvre de Janet Cardiff que le Musée d'art contemporain de Montréal a montée en 2002, et y est toujours disponible ${ }^{22}$.

formulations qui désignent cette structure dynamique: «going back and forth» (p. 21), «slipping in and out of » (p. 22, 29), «flip-flopping» (p. 27), « overlapping» (p. 78), ibid.

21. À titre d'exemples: Family Viewing d'Atom Egoyan (1987) et Alice de Marco Martins (2005).

22. C'est grâce à ce rattachement au MAC de Montréal que j’ai pu avoir un accès privilégié à la documentation de cette promenade, en particulier au script. Je tiens à remercier tous les représentants du musée qui m’ont très aimablement facilité cet accès. 
Peut-on raconter cette promenade? Difficilement, car, comme dans toutes les autres promenades, le récit est fragmenté, plusieurs scénarios possibles se superposent, de multiples pistes narratives sont ouvertes mais sans être menées à terme, des temporalités différentes se chevauchent, divers niveaux de réalité cohabitent, tout en nous interpellant avec la même intensité esthésique : la réalité du site réel, la bande vidéo enregistrée dans ce lieu, le soundscape réel et le bruitage de la bande sonore ${ }^{23}$, les diverses couches de souvenirs personnels, la mémoire collective du lieu où se serait dressé autrefois un orphelinat, la réalité onirique sous laquelle est placée toute la promenade. En fait, au tout début, une voix de femme nous annonce que, dans un rêve, elle a tué un homme. On voit alors une fille apporter la photographie d'un homme gisant par terre, sur le ventre. Pour une fraction de seconde, le corps de l'homme est situé dans le garage - où l'on passera plus tard - et on entend le cri d'une femme. Une tâche difficile pour qui voudrait démêler tout cela dans un récit qui devrait apporter de l'ordre et de la clarté, comme c'est par exemple prévu dans le programme du roman policier. Et pourtant les signes d'une quête de vérité se multiplient tout au long de la promenade :

- it's time to find out

- I try to find a connection : the telephone, the photograph, the hotel room

- try to figure it out

Le résultat de ce désir de savoir, de cette volonté de vérité ne nous sera jamais donné. Nous restons engagés dans une quête, en transit entre le chaos et la mise en ordre.

Un mot sur le site que Janet Cardiff a choisi pour cette promenade. Car chaque lieu réel qu'elle sélectionne est évidemment déjà investi de tout un potentiel de signification, si ce n'est d'une surdétermination historique et culturelle comme c'est le cas de la Villa Medici à Rome, des galeries souterraines sous la ville de Münster, de la station Liverpool de Londres, etc. Dans Conspiracy Theory, on est dans un paysage urbain qui est structuré en trois lieux différents, car on va du haut lieu de culture qu'est le Musée au non-lieu anthropologique qu'est le garage souterrain (sous la Place des Arts), en passant par les passages souterrains de la Place des Arts. En se référant à ces passages souterrains, le texte anglais a recours au mot arcades qui est le terme utilisé pour traduire le

23. Ayant fait la promenade trois fois, je me suis, en fait, trompé à plusieurs reprises en ce qui a trait au niveau de réalité à attribuer aux sons perçus. Plusieurs fois, je me suis retourné en réagissant à des pas derrière moi - qui s'avéraient n'exister que dans la réalité créée par la bande sonore. 
Passagen-Werk de Walter Benjamin. Nous nous trouvons donc doublement dans des lieux urbains qui connotent le «en transit» de la société moderne: d'abord les passages commerciaux rendus célèbres par Benjamin - version montréalaise cependant, puisque souterrains -, ensuite les «non-lieux» que l'anthropologue Marc Augé a fait connaître en y voyant l'emblème même de ce qu'il appelle la surmodernité. La chambre d'hôtel dans laquelle la bande vidéo nous déplace brièvement au début de la promenade renchérit encore sur ce cumul des lieux du «en-transit» puisque l'hôtel, également, est traditionnellement associé à la locomotion et connote donc pareillement voyage et migration qui renvoient à une forme moderne de nomadisme.

Il s'agit d'autant de lieux publics urbains, connotant le passage, où, en fait, promeneurs, nous ne faisons que passer. Ce sont des lieux publiquement accessibles, mais ce ne sont pas des lieux «ethnographiques» comme un théâtre, ou une salle de cinéma, ou une école où les personnes présentes simultanément forment une communauté, ne fût-ce que temporairement. Ici aussi nous croisons des passants, nous sommes co-présents, mais anonymement, sans nous connaître ni nous parler. Nos vies et histoires de vie ne font que se croiser, par hasard là, au moment de la promenade que nous a fait faire Janet Cardiff ${ }^{24}$. Cela participe de la contingence introduite dans l'œuvre d'art comme un matériau signifiant, et cela fait réfléchir sur ce qu'est la communauté, mais dans cette esthétique «en transit» cela ne fonde pas une communauté comme celle de la salle de concert, par exemple.

\section{L'EFFET FRIEDRICH}

Puisque Conspiracy Theory est une promenade qui ajoute la caméra vidéo à l'effet audio dont j’ai déjà parlé, je m’attarderai maintenant à la construction visuelle de l'expérience esthétique proposée par Janet Cardiff. Nous avons vu qu'elle prend grand soin, technologiquement et conceptuellement, de construire l'effet auditif. Trouverons-nous une construction équivalente de l'effet visuel? Il me semble que oui. Je crois avoir découvert dans cette promenade un dispositif d'énonciation visuel qui correspond à l'effet d'envoûtement que produit la bande

24. Une fois, j’ai été interpellé par un passant qui, rendu curieux par ces espèces de cyborgs se promenant avec écouteurs et caméra, m’a demandé ce que faisaient toutes les personnes ainsi accoutrées. Je me suis alors senti pris dans une double contrainte, entre la politesse consistant à répondre à cette interpellation imprévue (venue concrétiser la part de contingence qui fait partie du projet de Janet Cardiff) et l'obligation de suivre le rythme de la bande audio-visuelle pour ne pas perdre le fil programmé de la promenade. 
sonore. Pour la faire apparaître, je me permets d'ouvrir ici encore une digression sur l'histoire de l'art. Plus particulièrement, je rappelle une série de tableaux du peintre romantique allemand Caspar David Friedrich.

Je soutiendrai que ces tableaux ont en commun une même structure d'énonciation visuelle. Ce sont, pour la plupart, des tableaux de paysages naturels. Dans chaque tableau, nous trouvons une ou deux, rarement plusieurs, personnes représentées de dos, en général placées au premier plan. Ces personnes-silhouettes font l'objet de notre regard tout en regardant le paysage représenté par le peintre. Nous voyons ces personnages, nous pouvons les identifier selon leurs habits, selon leur identité générique révélée dans le titre du tableau (Wanderer ${ }^{25}$, Frau ${ }^{26}, \mathrm{Mann}^{27}$ ), mais leur traitement pictural ne nous permet pas vraiment de les connaître, puisque nous les voyons en silhouette la plupart du temps et que nous ne voyons pas leur visage, le lieu d'identification visuelle humaine par excellence ${ }^{28}$. Ce sont des personnes assurant surtout la fonction de relais de notre regard par rapport au paysage peint.

C'est par l'intermédiaire de ces personnes-silhouettes que nous sommes attirés et placés dans le tableau. Il y a une espèce d'identification visuelle qui a lieu. Ce sont comme des Leerstellen ${ }^{29}$ visuelles qui nous invitent à les occuper, à prendre la place disponible, aménagée par eux au premier plan du tableau. Mais il ne s'agit pas d'un mécanisme de pulsion scopique, mécanisme qui est à la base du voyeurisme et qui nous pousse à vouloir voir ce qu'il y a dans un espace interdit à notre regard. Dans ces tableaux, rien n'est vraiment interdit à notre regard. Il s'agit bien plutôt d'un dispositif d'énonciation visuel. Et des plus efficaces.

Nous n'avons pas non plus affaire à la figure du «montreur» qui établit une médiation entre le spectateur externe au tableau et l'objet représenté dans le tableau. Cette figure que nous trouvons dans bien des tableaux classiques, souvent dans l'espèce de contorsion que lui impose sa fonction qui consiste à faire le lien visuel entre l'extérieur du tableau (établissant souvent un contact oculaire avec le spectateur) et l'intérieur du tableau (la main pointée vers la scène

25. Der Wanderer über dem Nebelmeer (1819).

26. Frau vor untergehender Sonne (1818) ou encore Frau am Fenster (1822).

27. Zwei Männer in Betrachtung des Mondes (1819).

28. Comme le montre, dans un contexte bien différent, l'usage de la photo de passeport.

29. Voir la théorie littéraire des Leerstellen (lieux d'indétermination), que Wolfgang Iser a développée à partir de la phénoménologie littéraire de Roman Ingarden et qui désigne des vides que la concrétisation du texte littéraire par l'acte de lecture viendrait remplir. 
ou le personnage représenté). La personne-silhouette de Friedrich est moins un véritable personnage du tableau que ne l'est le montreur, elle est une présence vide dessinant les contours de la place que je suis appelé à occuper dans le tableau pour voir le paysage représenté30.

Déjà, l'écrivain allemand Heinrich von Kleist avait reconnu la radicalité et l'efficacité de ce dispositif d'énonciation visuel. Plus que cela: sa violence. Dans un commentaire de 1812, publié dans les Berliner Abendblätter, il parle de la capacité qu'ont les tableaux de Friedrich d'aspirer et d'absorber le spectateur dans la réalité représentée et de favoriser de la sorte l'abolition de la différence entre représenté et représentant qui fonde l'art mimétique. Il choisit, pour exprimer cette particularité de la peinture de Friedrich, une expression métaphorique qui se réfère au fonctionnement physiologique de l'œil:

... et comme [l'image], dans son uniformité et son étendue sans bornes, n’a de premier plan que le cadre, c'est comme si, à la regarder, on avait les paupières amputées $^{31}$.

L'image de Friedrich - grâce à l'absence de premier plan, sauf la personnesilhouette $^{32}$ - aurait donc la force de happer le spectateur visuellement, comme s'il avait les paupières coupées, comme si l'œil était privé de ses protections contre l'interpellation esthésique ${ }^{33}$. Pour résumer cet état de choses assez complexe je parlerai, pour le dire succinctement, d'un «effet Friedrich ", effet qui consiste en une absorption et identification :

Thus the work of art is denied the possibility of referring to something which is not itself. Instead a hermetic relation is established between the work of art and its beholder, who sees himself absorbed into the picture. [...] The picture has shortcircuited its traditional poles. It has become a medium of identity ${ }^{34}$.

30. C'est l'équivalent, mais en sens inverse, de l'ovale vide que le visage du touriste viendra remplir pour se faire photographier avec l'image cliché d'un site touristique.

31. Texte original: « $\ldots$ und da [das Bild] in seiner Einförmigkeit und Uferlosigkeit, nichts als den Rahmen zum Vordergrund hat, so ist es, wenn man es betrachtet, als ob Einem die Augenlieder (sic) weggeschnitten wären» (Heinrich von Kleist, Berliner Abendblätter, 13 octobre 1812, $\mathrm{n}^{\circ}$ 12).

32. J'insiste davantage sur le rôle de la personne-silhouette vue de dos que Kleist et Kroeber.

33. Contrairement à l'oreille, l'œil est doté d'un mécanisme physiologique de protection.

34. Jörg Träger, «Imagination in Turner, Friedrich, and David», Frederick Burwick et Jürgen Klein (dir.), The Romantic Imagination. Literature and Art in England and Germany, Amsterdam, Rodopi, 1996, p. 416-417. 
En sens inverse, ce dispositif d'énonciation visuel produit exactement l'accès direct à l'intériorité du sujet de l'expérience esthétique que Janet Cardiff a attribué à la perception sensorielle de l'ouie. Friedrich aurait en quelque sorte découvert une stratégie qui contourne les mécanismes de filtrage et de défense dont disposerait le sens de la vue. Il sait forcer l'ouverture des yeux, comme si les paupières étaient amputées. L'image de Kleist, étonnamment, anticipe ainsi métaphoriquement ce que la fiction cinématographique a réalisé de nos jours. Je pense aux scènes de torture, la plus connue étant celle de Clockwork Orange (1971) de Stanley Kubrick, où l'on ouvre de force les yeux d'un individu pour qu'il voie ce qui est insupportable à voir. C'est le même genre de torture que subit le protagoniste dans le film qui porte le même titre que la promenade de Janet Cardiff: Conspiracy Theory.

Janet Cardiff n'est pas allée jusqu'à des images de torture physique. Mais elle a recours à «l'effet Friedrich» pour mettre la vue à égalité avec l'ouïe quant au pouvoir de transfert imaginaire identificatoire. Elle se sert d'une figure féminine - une femme habillée d'une robe blanche et aux longs cheveux noirs - qui apparaît une demi-douzaine de fois dans la bande vidéo et que nous ne voyons pratiquement que de dos:

- nous la voyons de dos à travers la fenêtre de la chambre d'hôtel;

- elle s'éloigne en courant dans le flash du corps de l'homme étendu à terre;

- nous la retrouvons dans le passage (les arcades) à l'extérieur du musée;

- elle se tient devant nous, encore de dos, juste avant l'arrivée, à toute vitesse, d'une voiture dans le garage;

- finalement, à deux reprises, elle court devant nous, à toutes jambes, dans les passages souterrains de la Place des Arts.

Toujours la même femme mystérieuse - nous ne connaissons guère son visage - qui se tient devant nous de dos. Comme chez Friedrich, c'est la figure-relais qui nous entraîne dans l'histoire, nous désirons nous situer à sa place, adopter son regard pour savoir ce qui se passe, ce qui s'est passé. Un effet de transposition-identification involontaire se produit. Évidemment, par rapport à la peinture, s'y ajoute la dynamique du mouvement.

Si l'on superpose ce que je viens de développer au sujet de la bande vidéo aux effets sonores et aux structures d'énonciation verbale (dissociation «I»/«you» <=> coalescence «we »), on constate qu'une même dynamique paradoxale d'immédiateté par médiation est à l'œuvre dans le sonore et dans le visuel. Un même contact immédiat-mais-médiatisé a lieu: immédiat parce qu'on est transposé dans l'autre type de réalité et qu'un effet d'identification s'ensuit avec diverses instances de la narration; médiatisé parce que tous ces processus sont 
produits et obtenus par un usage artistique des plus raffinés des médias et des technologies les plus sophistiqués.

Là où Janet Cardiff nous entraîne un cran plus loin que Friedrich dans l'application de son dispositif visuel d'énonciation, c'est quand elle le dynamise en le prolongeant dans une série potentiellement infinie: nous marchons derrière cette femme-silhouette mystérieuse, nous sommes entraînés dans son histoire, et nous aimerions voir devant elle à travers ses yeux là où il y a potentiellement quelqu'un qui marche, vu de dos... Derrière nous, nous entendons des pas; quelqu'un nous suit, nous voit de dos... Et la voix de suggérer «there is somebody behind you » ou encore «we are being watched ${ }^{35}$ ». Ou encore, voici le résumé de l'effet Friedrich mis en abîme dans le texte parlé de la promenade de Münster: «He follows her, just as I follow him, wanting to see through her eyes, to think her thoughts ${ }^{36}$. » Il y a un passage dans Conspiracy Theory où le texte parlé (dit par Janet Cardiff) rend ce processus de substitution explicite, tout en le complexifiant d'une relation temporelle entre présent et passé. C'est au moment où, dans les passages souterrains, on retrouve la femme mystérieuse:

There she is again. Turn to the right. Follow her.

I remember now, walking here the first time. I didn't know they were following behind me like this... shooting me with a camera. I watch my own image now, but it's like she's another woman.

Ceci nous ramène à la matrice même de la structure de l'expérience esthétique que nous offre Janet Cardiff tout en la construisant minutieusement, grâce à des appareils technologiques du dernier cri. Le mouvement s'ouvre en amont et en aval, la transposition s'articule en série, rien n'est plus stable, aucun aboutissement, aucune ultime référence (de vérité, de réalité, d’identité...) ne s'annonce comme instance de stabilisation. Tout reste en mouvement, l'expérience esthétique n'offre que passage, transition, bref une expérience en transit.

\section{L'EXPÉRIENCE ESTHÉTIQUE QUI DONNE À PENSER}

Je pourrais m'arrêter ici, ayant montré comment l'activité artistique de Janet Cardiff reprend et interagit avec les conditions de la culture contemporaine en combinant locomotion avec médiamotion dans un genre artistique tout à

35. On peut même ajouter à cette série les diapositives que Janet Cardiff a «autorisées » pour documenter sa promenade : est photographiée, de dos, une personne qui est en train de faire la promenade et donc en train de suivre la femme en robe blanche qu'elle ne peut voir que de dos!

36. Christov-Bakargiev, 2001, p. 89. 
fait original, et comment cette forme artistique détermine l'expérience esthétique particulière du promeneur de ses walks. J'aurais ainsi montré combien elle appartient à son temps et utilise les moyens et matériaux offerts par son époque de la manière la plus créative. Mais conclure ainsi ne ferait d'elle qu'une artiste largement tributaire de son temps, un témoin-artiste de notre condition culturelle.

Or, il y a plus - Janet Cardiff pousse son travail d'artiste plus loin dans ses promenades. Car, tout en travaillant avec des sites réels d'aujourd'hui, et en se servant de matériaux que le développement technologique le plus récent a mis à sa disposition, elle aménage dans ses œuvres, et par là dans l'expérience esthétique de ses promeneurs, un espace de réflexion sur les conditions culturelles environnantes. Tout en plongeant, avec l'intensité décrite et analysée, son promeneur dans une immédiateté esthétique des plus prenantes, elle réussit à nous aménager une position de surplomb par rapport à ces conditions mêmes. C'est dans cet espace additionnel, dans ce surplomb que vient s'inscrire la composante épistémique et critique de l'œuvre. Je l'illustrerai à l'aide de deux exemples tirés de Conspiracy Theory.

Lutilisation esthétique des médias et technologies les plus modernes par l'artiste est en fait doublée d'une seconde présence de ces mêmes médias et technologies dans la promenade: ils sont thématisés, font l'objet d'une monstration, ce qui nous permet de les voir ${ }^{37}$ - pas seulement de voir et d'entendre à travers eux -, d'abord, et de les interroger ensuite quant à leur présence, utilité et effets pervers. Cette monstration ouvre un espace de réflexion qui est aménagé par l'artiste pour son promeneur, et ceci en vertu d'un certain détachement de l'expérience esthétique par rapport à l'expérience tout court, quels que soient par ailleurs le chevauchement et l'imbrication voulus que Janet Cardiff autorise entre les deux types d'expérience. Un tel espace de réflexion n’est pas aménagé dans l'expérience quotidienne, il faut le créer, presque l'arracher au quotidien par toutes sortes d'astuces et de tactiques, si tant est qu'on y tienne et ne se contente pas d'une attitude fataliste-consumériste.

En fait, dans Conspiracy Theory, Janet Cardiff nous «montre» un grand nombre de médias, de plusieurs générations. Ils sont intégrés dans le déroulement diégétique de la promenade, tout en s'en détachant légèrement comme par des gestes brechtiens de distanciation (Verfremdung). Pour commencer, la caméra qu'on utilise est sans cesse nommée, identifiée comme un instrument de quête

37. J'utilise le verbe «voir» ici, dans la bonne tradition «oculocentriste» comme désignant à la fois un acte de perception corporelle et un acte cognitif et intellectuel. 
et non pas comme un œil technique qui donnerait mieux accès à la réalité : hold the camera up, point the camera where I point it. De toute façon, nous ne voyons pas la réalité à travers la caméra, nous voyons, grâce à la caméra numérique, sur le moniteur, le résultat d'une mise en scène filmée sur les mêmes lieux. Cette image vidéo contribue à notre schizophrénie perceptive et génère de la sorte une dissonance cognitive qu'il nous revient de résoudre. Elle ne se réduit jamais à la fonction d'instrument neutre d'enregistrement visuel pour le promeneur. Elle est à la fois indispensable et problématique. De plus la voix la relie aux caméras de surveillance du Musée, en rappelant que le même appareil assume, en même temps, sur les mêmes lieux, d'autres fonctions que celles de nous guider dans une expérience esthétique.

Un traitement analogue est réservé à la photographie, au moyen de laquelle nous connaissons visuellement la victime présumée d'un meurtre, comme une pièce à conviction, une preuve extra-discursive dans un procès. Le téléphone intervient dans une double version : il y a, d'une part, le cellulaire qu'utilise le personnage attablé dans le restaurant du Musée et, d'autre part, la batterie de téléphones fixes aménagée dans les passages souterrains de la Place des Arts. Les deux font entièrement partie de l'aspect de médiamotion que comporte cette promenade, en ouvrant des espaces imaginaires extérieurs à celui qui est accessible à nos sens immédiats. Comme nous n'entendons jamais le contenu des conversations téléphoniques, le téléphone, dans l'atmosphère du complot, devient un générateur de suspicion. Il en est de même d'une vidéocassette trouvée sur le lieu présumé du crime, et des documents portés dans une sacoche par un inconnu, également trouvés dans une enveloppe à même le sol.

La présence de tous ces appareils techniques et médias constitue un trait « réaliste » qui ajoute à l'effet de vraisemblance du récit et opère en même temps une mise en abîme réflexive sur la mobilité médiatique dans laquelle nous vivons et sur laquelle s'appuie la réalisation technique de cette promenade.

L'autre exemple est l'usage que Janet Cardiff fait de la locomotion dans les promenades. Il est certain que l'élément de locomotion qu'elle intègre ici est à la base de l'originalité du genre artistique qu'elle a développé. Peu d'expériences esthétiques plus traditionnelles (par exemple lire un livre, aller au concert, aller au musée, contrairement peut-être à voir une statue, un monument architectural) comportaient constitutivement ce déplacement physique dans un lieu réel. Et il me paraît certain que cette invention esthétique est à relier à la mobilité ambiante de notre société et culture. Mais, si elle reprend ainsi un aspect de la condition de vie contemporaine, elle s'en détache aussi, en y introduisant une différence importante. 
C'est que, par rapport au volume et à la vitesse technologique des déplacements et des migrations qui caractérisent notre monde réel, la déambulation ramène en quelque sorte la locomotion à un degré zéro anthropologique. Elle y introduit deux paramètres qui peuvent être interprétés comme contribuant à une esthétique de la résistance par rapport au monde environnant: tout d'abord, son expérience subjective individuelle créant une distance critique par rapport à tout mouvement de masse; ensuite, sa lenteur irrévocable ${ }^{38}$ : l'expérience physique de mon déplacement déambulatoire, avec son rythme lent qui est artistiquement programmé, constitue un véritable îlot de lenteur. En contraste avec la vitesse offerte et imposée par les moyens de transport modernes, le ralentissement qu'opère la promenade dans le rythme de nos vies quotidiennes - surtout urbaines - opère une différence significative. Janet Cardiff, par ses promenades artistiques, nous invite à faire une expérience de locomotion inédite, à entrer, esthétiquement, dans une autre vitesse et une autre temporalité propices à une réflexion critique. Je crois que c'est par cette lenteur de la déambulation que l'expérience esthétique des promenades nous donne le plus à penser. Et c'est par là aussi que Janet Cardiff contribue à un discours critique à circulation internationale qui prend la forme d'un éloge de la lenteur ${ }^{39}$, et qu'elle participe, aux côtés du dramaturge Marthaler, ou des cinéastes Tarkovski et Chris Marker, d'une esthétique de la lenteur qui proteste contre une certaine tyrannie du temps et de la vitesse qui nous tient dans le quotidien ${ }^{40}$.

38. Il n'y a pas moyen d'accélérer, de comprimer l'expérience que Janet Cardiff nous propose de faire dans ses promenades, comme on peut le faire d'une lecture, d'une visite au musée ou d'un monument, voire du visionnement d'un film en format DVD.

39. Reproduit en France, par exemple, par Jacques Réda et par Pierre Sansot.

40. Voir Fritz Reheis, Die Kreativität der Langsamkeit. Neuer Wohlstand durch Entschleunigung, Darmstadt, WBD, 1996. 